\title{
An intermodal multicommodity routing problem with scheduled services
}

\author{
Burak Ayar · Hande Yaman
}

Published online: 9 June 2011

(C) Springer Science+Business Media, LLC 2011

\begin{abstract}
We study a multicommodity routing problem faced by an intermodal service operator that uses ground and maritime transportation. Given a planning horizon, a set of commodities to be picked up at their release times and to be delivered not later than their duedates, the problem is to decide on routes for these commodities using trucks and scheduled and capacitated maritime services at minimum cost of transportation and stocking at the seaports. Two mixed integer programming formulations and valid inequalities are proposed for this problem. The results of a computational study to evaluate the strength of the linear programming relaxations and the solution times are reported.
\end{abstract}

Keywords Intermodal routing $\cdot$ Multicommodity flows $\cdot$ Scheduled services $\cdot$ Valid inequalities

\section{Introduction}

Intermodal transportation can be broadly defined as the transportation of goods or people using more than one transportation mode, in an integrated manner from an origin to a destination (for more definitions, we refer the reader to Bontekoning et al. [6]). In this paper, we use this term to refer to the transportation of containerized goods by a same company using a mix of truck, rail, air and/or maritime transportation.

Even though some earlier examples are cited, the tremendous increase of intermodal transportation has followed the use of standardized containers (see, e.g., Slack

B. Ayar $(\bowtie) \cdot$ H. Yaman

Department of Industrial Engineering, Bilkent University, 06800 Ankara, Turkey

e-mail: ayar@bilkent.edu.tr

H. Yaman

e-mail: hyaman@bilkent.edu.tr 
[19]). The standardization in dimensions made it possible to use tools for loading and unloading at the intermodal terminals and hence decreased the complexity and the cost of these operations. The trend of increase has been amplified by the growth in international trade in the last decades. The limitations posed, and the incentives and subsidies given by governments with the aim of decreasing the ground traffic have also contributed to the increase in volumes carried using different transportation modes.

In this paper, we study a multicommodity network flow problem faced by a third party logistics company. The company uses ground and maritime transportation. We are given a set of commodities, which should be picked up from their origins at given release times and should be delivered to their destinations no later than their duedates. The commodities may be carried directly from their origins to their destinations on trucks or they may be carried on trucks to a seaport, may visit several seaports using maritime services, and then be carried to their destinations on trucks. As the company rents trucks, there is no capacity and time limitation on the use of ground transportation. However the maritime services are scheduled in advance and the company has limitations on the amounts of volume that it can carry on each service. Each maritime service has a start time of loading and a cutoff time after which loading is not permitted. For a commodity to be able to use a service, it should be available at the origin seaport of the service before the cutoff time of this service. If a commodity arrives at the origin seaport too early compared to the start of loading, then a cost is incurred for stocking at the seaport. The aim is to determine routes for commodities in order to minimize the total cost of shipments over a given time horizon, respecting the capacity and time related constraints. The total cost has two components: the transportation cost and the stocking cost at seaports. We call this problem the "Intermodal Multicommodity Routing Problem with Scheduled Services (IMR-S)".

Even though the study is motivated by an application in the context of freight logistics and maritime transportation, the problem applies to more general intermodal transportation settings, where some services are scheduled in advance of the routing decisions. For instance, a company selling travel packages may need to route groups of people from some origins to destinations using buses, which may be rented and thus are available at any time, together with train and plane trips, which have fixed schedules and on which the number of seats reserved to the company is limited. If the travelers need to wait for long time periods before taking a vehicle, there may be a need to provide accommodation at some additional cost. Even in cases where this is not necessary, such long waiting times may decrease customer satisfaction. Hence, it is reasonable to associate some cost for waiting times between transfers. Now, the problem of finding time and capacity feasible and minimum cost routes for groups of travelers can be modeled as $I M R-S$.

In this paper, we first investigate the computational complexity of our problem and prove that it is NP-hard. Then we seek strong and small size formulations for IMR-S. In the literature, similar problems are usually modeled using time-space networks. As these networks expand the underlying transport networks with a time dimension, they have large sizes. As a result, the formulations based on these networks use a large number of variables to model routing decisions. Here, we propose a first mixed integer programming formulation without resorting to a time-space network representation and strengthen it using variable fixing and valid inequalities. Next, we relax the 
capacity constraints in a Lagrangian manner and show that the relaxed problem decomposes into a series of shortest path problems defined on networks augmented by time for each commodity. The corresponding Lagrangian dual yields a lower bound, which may be stronger than the one of the linear programming relaxation of our first formulation. Then we provide an extended formulation, a formulation based on a time-space network representation, whose linear programming relaxation gives the same bound as the Lagrangian dual. The time-space network that forms the basis for this formulation has a polynomial size in the size of the original service network. Finally, we report the results of our computational study where we compare the strength and solution times for these two formulations. We observe that our first formulation strengthened with the valid inequalities outperforms the time-space network based extended formulation, even though the latter formulation is stronger and the size of the time-space network grows polynomially with the size of the original network. Hence our main contribution is the derivation of this smaller size formulation and the associated valid inequalities, which enable us to solve larger instances for which the extended formulation based on the time-space network representation is computationally not efficient.

Below, we first mention some important survey papers and then we summarize the work done in the field of intermodal routing.

Bontekoning et al. [6] and Macharis and Bontekoning [15] survey the inland intermodal freight transportation literature. Macharis and Bontekoning [15] classify the studies in this field using two criteria: type of operator and time horizon of operations. They identify four types of operators, namely, drayage, terminal, network, and intermodal operators, and three planning levels, namely, strategic, tactical and operational. Our problem is an operational level planning problem faced by an intermodal service operator according to this classification and there are only a few studies in this category.

Crainic and Kim [11] survey the literature on problems encountered in operating a container-based intermodal transportation network. A survey of the studies on cargo shipping and planning problems in maritime transportation is given by Christiansen et al. [10]. Bektas and Crainic [4] give an overview of intermodal transportation with the shipper and the carrier perspectives and discuss several problems encountered in service network design, operational planning, and intermodal terminals. Pedersen [18] summarizes the development of intermodal transportation in Europe, outlines important optimization problems encountered in this domain, and studies service scheduling problems.

Barnhart and Ratliff [2] consider the problem of transporting commodities on an uncapacitated rail-truck network with the aim of minimizing the total transportation and inventory costs. When the transportation cost is charged on a per trailer basis, the problem decomposes into a set of shortest path problems, one for each commodity. However, when the charge is on a per flatcar basis, the problem is modeled as a matching problem under the assumption that each flatcar can transport at most two trailers. Extensions to incorporate duedates and restrictions on flatcar configurations are discussed. A chance constrained goal programming model to determine intermodal routes to minimize cost and risks is given by Min [16]. On time service requirements are included in this model. Boardman et al. [5] describe a decision sup- 
port system to choose the least cost combination of transportation modes on an intermodal network. Bookbinder and Fox [7] study the problem of finding optimal intermodal routes for Canada-Mexico shipments under NAFTA. Intermodal alternatives on a network connecting five major Canadian cities and three major Mexican cities are evaluated and nondominated solutions with respect to total time and total cost objectives are identified. Kim et al. [14] consider the problem of determining the flow and the number of vehicles of each transportation mode on a network in order to minimize the sum of shipping and transportation costs under capacity and vehicle restrictions. They derive a mixed integer programming model and present a case study based on the container cargo data in Korea. Ziliaskopoulos and Wardell [22] present an algorithm to compute intermodal time-dependent least travel time paths. The algorithm takes into account the delays during the change of modes and the fixed schedules. Boussedjra et al. [8] develop an exact method to find the least travel time path between an origin destination pair in a time-dependent intermodal transportation network. They use the number of transshipments as a second criterion if there exists a tie between two solutions. Song and Chen [20] consider the problem of finding a least cost path between an origin destination pair with a delivery time limit on an intermodal network with scheduled services. The total cost is the sum of transportation cost, the transition cost, and the holding cost. They show that the problem can be modeled as a shortest path problem on a time-space network. Grasman [13] presents dynamic programming approaches to compute minimum cost path with on time service requirements and minimum lead time path subject to cost restrictions for intermodal networks.

The two closest studies to our problem are the ones by Chang [9] and Moccia et al. [17]. Chang [9] studies the problem of determining routes of commodities on international intermodal networks. The problem is a multiobjective intermodal multicommodity flow problem with time windows and concave costs. The objectives are minimizing the total transportation cost and the total travel time. The transportation cost is a concave piecewise linear function of total flow. A time window is associated with each node to define the earliest and latest arrival times to the node in order to use the services originating from this node. Both scheduled and time-flexible services are considered. A commodity departs from a node after its service time or leaves the node at a scheduled departure time if it is using a scheduled service. The demand of a commodity may be split among several paths. This problem is converted into a single objective problem by taking a weighted sum of the objectives. Then Lagrangian relaxation is applied to decompose the problem. A heuristic to construct feasible solutions from the solutions of the relaxed problem is presented. Finally the method is tested on a small illustrative example and a larger network.

The problem studied by Moccia et al. [17] generalizes the problem in Chang [9] by considering multiple time windows for pickup and deliveries and general (not necessarily concave or convex) piecewise linear transportation costs. However, a single objective, the one of minimizing the total transportation cost, is considered and single path routing is imposed as a constraint in this study. The authors describe how to represent their problem using a directed network where multiple copies of nodes are created and some additional nodes are introduced to be able to model the multiple time windows and scheduled departure times. Then two formulations, an arc-node 
and a path formulation, are given along with valid inequalities. Column generation algorithms are designed to obtain lower bounds and are combined with heuristics to get feasible solutions. The methods are tested on data obtained from an Italian freight forwarder that uses rail and road transportation.

Our problem can be seen as a particular case of the problem that is studied by Moccia et al. [17], where pickup time windows have lower and upper limits equal to the release times and the delivery time windows are equal to the intervals starting at the release times and ending at the duedates. Different from the problem of Moccia et al. [17], we include penalty costs for waiting times spent at the seaports. However, it is possible to handle these costs with the approach presented in Moccia et al. [17]. The approach we present here is tailored for our special case.

The paper is organized as follows. In Sect. 2, we give a detailed definition of our problem IMR-S and prove that it is NP-hard. A first mixed integer programming formulation is given in Sect. 3. In Sect. 4, we present results on variable fixing and valid inequalities. Section 5 presents a Lagrangian relaxation and an extended formulation derived using this relaxation. The results of a computational study comparing the first model and the extended model and investigating the use of valid inequalities are reported in Sect. 6. The paper is concluded with further research directions in Sect. 7.

\section{Problem definition and complexity}

Here, we first define our problem more formally and then establish its computational complexity. We are given a directed network $G=(N, A)$, which possibly contains parallel arcs. Each node in this network corresponds to a seaport and each arc corresponds to a scheduled maritime service. For service $a \in A, s_{a}$ denotes the origin seaport, $t_{a}$ denotes the destination seaport, $\left[e_{a}, l_{a}\right]$ denotes the time window $\left(e_{a}\right.$ is the start time of loading and $l_{a}$ is the cutoff time), $c_{a}$ denotes the cost of transporting a unit volume, $\tau_{a}$ denotes the travel time, and $u_{a}$ denotes the capacity of service $a$.

We denote by $p_{i}$ the cost of stocking a unit volume at seaport $i \in N$ for a unit time.

The set of commodities is denoted by $K$. A commodity $k \in K$ has origin $o_{k}$, destination $d_{k}$, demand volume $w_{k}$, release time $r_{k}$, and duedate $q_{k}$.

For $k \in K$, let $c_{o_{k} d_{k}}$ denote the unit cost of transporting commodity $k$ from its origin to its destination directly using trucks and $\tau_{o_{k} d_{k}}$ denote the corresponding travel time. For $k \in K$ and $i \in N$, let $c_{o_{k} i}$ and $\tau_{o_{k} i}$ denote the unit cost and time for carrying commodity $k$ from its origin to seaport $i$, and $c_{i d_{k}}$ and $\tau_{i d_{k}}$ denote the unit cost and time for carrying commodity $k$ from seaport $i$ to its destination directly using trucks, respectively.

For a given commodity $k \in K$, a time feasible intermodal route is a simple path that starts at the origin $o_{k}$ at time $r_{k}$, visits a set of seaports using a set of maritime services respecting the cutoff times, and ends at the destination $d_{k}$ not later than the duedate $q_{k}$. The cost of such a route is the sum of routing and waiting costs. For each commodity, we decide either to route the commodity directly on trucks or to route it on a time feasible intermodal route respecting the capacities of maritime services with the aim of minimizing cost. 


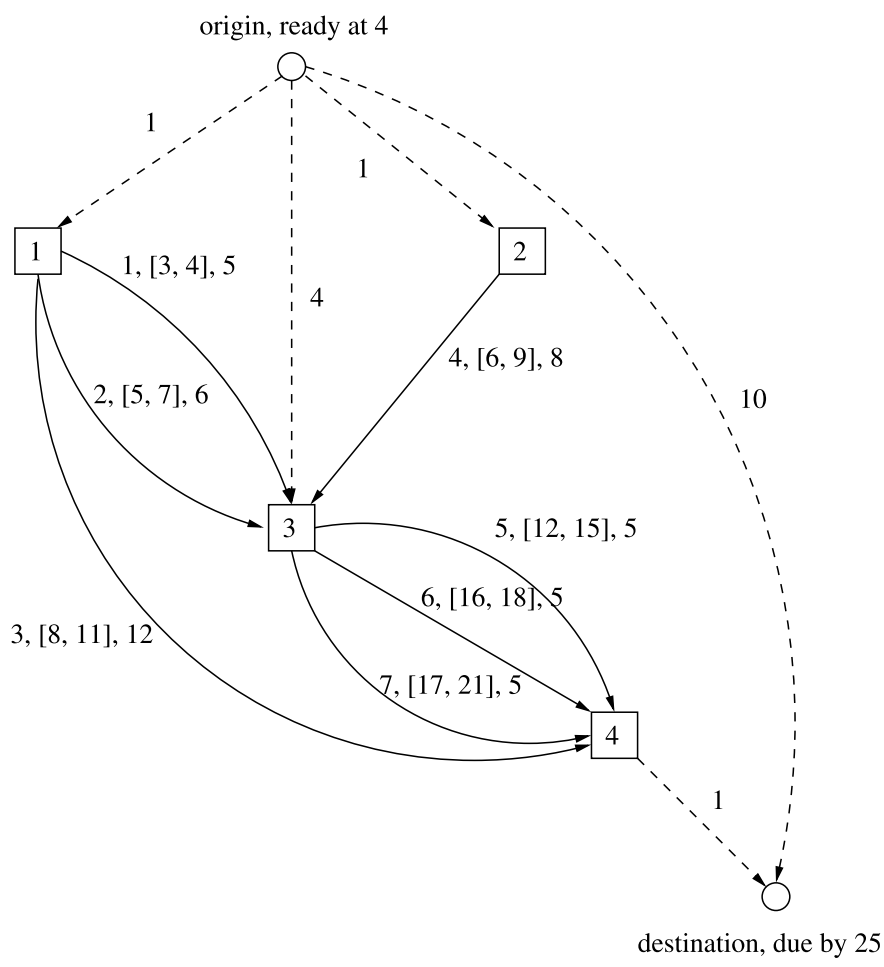

Fig. 1 An example service network

We provide an example in Fig. 1 to clarify the definitions. Here, we have four seaports and seven maritime services. Hence, $N=\{1, \ldots, 4\}$ and $A=\{1, \ldots, 7\}$. We consider a single commodity whose release time is 4 and duedate is 25 . Maritime services are represented with solid lines and truck connections are represented with dashed lines. To keep the example small, we assume that truck connections from the origin to seaport 4 and from seaports 1,2, and 3 to the destination are not available. The numbers on truck connections are the corresponding travel times. For each maritime service, we give the number of the service, the time window [the start time of loading, the cutoff time], and the travel time. In our example, it is possible to transport the commodity from its origin to its destination using trucks in 10 days. It is also possible to go to seaports 1,2 , and 3 by trucks. If the commodity is transported to seaport 1 on trucks, it arrives there at time 5. It is not possible to use maritime service 1 as its cutoff time is equal to 4, which is earlier than the arrival time of our commodity. Maritime services 2 and 3 can be used. If service 2 is used, then the commodity is loaded without waiting, leaves the seaport 1 at time 7 and arrives at seaport 3 at time 13. On the other hand, if service 3 is used, then the commodity is stocked at the seaport 1 for 3 units of time until the start of loading. Then it leaves seaport 1 at time 11 and arrives at seaport 4 at time 23. Alternatively, the commodity can be transported using trucks to seaport 2, where it waits for 1 unit of time until the start of loading, leaves seaport 2 using maritime service 4 at time 9, and arrives at seaport 3 
at time 17. There are three maritime services leaving seaport 3 and arriving at seaport 4. As the cutoff time for service 5 is equal to 15 , it can be used only if we arrive at seaport 3 using direct truck connection from the origin or using maritime service 2. Maritime service 7 cannot be used, as it arrives at seaport 4 at time 26, which is later than the duedate. Overall, there are seven time feasible routes for our commodity:

1. direct truck connection from origin to destination (arrival time 14)

2. truck connection from origin to seaport 1, waits at seaport 1 for 3 units of time, maritime service 3 , truck connection from seaport 4 to destination (arrival time 24)

3 . truck connection from origin to seaport 1 , maritime service 2, maritime service 5, truck connection from seaport 4 to destination (arrival time 21)

4. truck connection from origin to seaport 1 , maritime service 2, waits at seaport 3 for 3 units of time, maritime service 6 , truck connection from seaport 4 to destination (arrival time 24)

5. truck connection from origin to seaport 2, waits at seaport 2 for 1 unit of time, maritime service 4 , maritime service 6 , truck connection from seaport 4 to destination (arrival time 24)

6. truck connection from origin to seaport 3, waits at seaport 3 for 4 units of time, maritime service 5, truck connection from seaport 4 to destination (arrival time 21)

7. truck connection from origin to seaport 3, waits at seaport 3 for 8 units of time, maritime service 6 , truck connection from seaport 4 to destination (arrival time 24).

We define the decision version of the problem $I M R-S$ as follows. Given the parameters of the problem and a scalar $\gamma_{0}$, does there exist a feasible solution with cost not more than $\gamma_{0}$ ? Next, we prove that this problem is NP-complete using a polynomial reduction from the $0-1$ knapsack problem.

\section{Proposition 1 The decision version of IMR-S is NP-complete.}

Proof The decision version of IMR-S is in NP. Consider the decision version of the 0-1 knapsack problem. Given a set $I$, nonnegative integers $\alpha_{i}$ and $\beta_{i}$ for each $i \in I$ and two positive scalars $\alpha_{0}$ and $\beta_{0}$, does there exist a subset $S \subseteq I$ such that $\sum_{i \in S} \alpha_{i} \leq \alpha_{0}$ and $\sum_{i \in S} \beta_{i} \geq \beta_{0}$ ? This problem is NP-complete (see Garey and Johnson [12] problem [MP9]) even when $\alpha_{i}=\beta_{i}$ for all $i \in I$.

Suppose that there are only two seaports 1 and 2 , and that there is a single service from 1 to 2 with capacity $\alpha_{0}$. For each item $i$ in set $I$, define a commodity from node 1 to node 2 with demand volume equal to $\alpha_{i}$. The release time is 0 and the duedate is 1 for all commodities. Commodities can be transported at no cost and no time from their origins to seaport 1 and from seaport 2 to their destinations. The maritime service starts at time 0 and ends at time 1 . The unit cost of using the service is equal to 1 and the unit cost of delivering a commodity using trucks is 2 . Under this specification of parameters, the $I M R-S$ reduces to the problem of finding a minimum cost partition of the set of commodities $I$ into two sets $S$ and $I \backslash S$ such that commodities $S$ are transported using the maritime service and commodities $I \backslash S$ are transported using trucks. Such a partition is feasible if $\sum_{i \in S} \alpha_{i} \leq \alpha_{0}$. The cost of the associated solution is $\sum_{i \in S} \alpha_{i}+\sum_{i \in I \backslash S} 2 \alpha_{i}=2 \sum_{i \in I} \alpha_{i}-\sum_{i \in S} \alpha_{i}$. Let $\gamma_{0}=2 \sum_{i \in I} \alpha_{i}-\beta_{0}$. 
Now there exists a solution to the 0-1 knapsack problem with $\beta_{i}=\alpha_{i}$ for all $i \in I$ if and only if there exists a solution to the decision version of the problem IMR-S.

\section{A mixed integer programming model}

In this section, we present a mixed integer programming model for IMR-S. In this model, we use the following decision variables. For $k \in K, y^{k}$ is 1 if commodity $k$ is transported directly from its origin to its destination on trucks and 0 otherwise. For $k \in K$ and $i \in N, x_{o_{k} i}^{k}$ is 1 if commodity $k$ is carried from its origin directly to seaport $i$ using trucks and 0 otherwise and $x_{i d_{k}}^{k}$ is 1 if commodity $k$ is carried from seaport $i$ to its destination directly using trucks and 0 otherwise. For $k \in K$ and $a \in A$, $x_{a}^{k}$ is 1 if commodity $k$ uses maritime service $a$ and 0 otherwise. Finally, for $k \in K$ and $i \in N, v_{i}^{k}$ denotes the arrival time of commodity $k$ at seaport $i$ if this seaport is visited by this commodity and $z_{i}^{k}$ is the amount of time for which commodity $k$ waits at the stocking area at seaport $i$.

Using the above decision variables, we derive the following mixed integer programming formulation called $M 1$.

$$
\begin{aligned}
& \min \sum_{k \in K} w_{k}\left(\sum_{i \in N} c_{o_{k} i} x_{o_{k} i}^{k}+\sum_{a \in A} c_{a} x_{a}^{k}+\sum_{i \in N} c_{i d_{k}} x_{i d_{k}}^{k}+c_{o_{k} d_{k}} y^{k}+\sum_{i \in N} p_{i} z_{i}^{k}\right) \\
& \text { s.t. } \quad \sum_{i \in N} x_{o_{k} i}^{k}+y^{k}=1 \quad \forall k \in K \\
& \sum_{a \in A: t_{a}=i} x_{a}^{k}+x_{o_{k} i}^{k}-\sum_{a \in A: s_{a}=i} x_{a}^{k}-x_{i d_{k}}^{k}=0 \quad \forall k \in K, i \in N \\
& \sum_{i \in N} x_{i d_{k}}^{k}+y^{k}=1 \quad \forall k \in K \\
& \sum_{a \in A: t_{a}=i} x_{a}^{k}+x_{o_{k} i}^{k} \leq 1-y^{k} \quad \forall k \in K, i \in N \\
& \sum_{k \in K} w_{k} x_{a}^{k} \leq u_{a} \quad \forall a \in A \\
& v_{i}^{k}=\sum_{a \in A: t_{a}=i}\left(l_{a}+\tau_{a}\right) x_{a}^{k}+\left(r_{k}+\tau_{o_{k} i}\right) x_{o_{k} i}^{k} \quad \forall k \in K, i \in N \\
& z_{i}^{k} \geq \sum_{a \in A: s_{a}=i} e_{a} x_{a}^{k}-v_{i}^{k} \quad \forall k \in K, i \in N \\
& v_{i}^{k} \leq \sum_{a \in A: s_{a}=i} l_{a} x_{a}^{k}+\left(q_{k}-\tau_{i d_{k}}\right) x_{i d_{k}}^{k} \quad \forall k \in K, i \in N \\
& r_{k}+\tau_{o_{k} d_{k}} y^{k} \leq q_{k} \quad \forall k \in K \\
& x_{o_{k} i}^{k}, x_{i d_{k}}^{k} \in\{0,1\} \quad \forall k \in K, i \in N
\end{aligned}
$$




$$
\begin{aligned}
& x_{a}^{k} \in\{0,1\} \quad \forall k \in K, a \in A \\
& y^{k} \in\{0,1\} \quad \forall k \in K \\
& z_{i}^{k} \geq 0 \quad \forall k \in K, i \in N .
\end{aligned}
$$

Constraints (2)-(4), (12), and (13) ensure that either a commodity is transported from its origin to its destination directly using trucks or there exists a path from its origin to its destination that visits only seaports on its way. Constraints (5) eliminate cycles. First observe that there always exists an optimal solution without any cycle that does not intersect the path of a commodity, since costs are nonnegative. It is also not possible to have a cycle that starts and ends at the origin or destination of a commodity. Hence it is sufficient to ensure that each commodity can arrive at a seaport at most once to eliminate cycles.

Constraints (6) ensure that total volume to be transported on a service cannot exceed the capacity of the service.

Constraints (7) compute the arrival times of commodities to seaports. If commodity $k$ arrives at seaport $i$ using service $a$, then it leaves the origin seaport $s_{a}$ at time $l_{a}$ and travels for $\tau_{a}$ time units, and hence arrives at $i$ at time $l_{a}+\tau_{a}$. If seaport $i$ is the first seaport that commodity $k$ visits, i.e., if commodity $k$ leaves its origin and comes to $i$ using trucks, then $r_{k}$ is the time trucks leave the origin node and $\tau_{o_{k} i}$ is the trip time. Hence, the arrival time is $r_{k}+\tau_{o_{k}}$. If commodity $k$ does not visit seaport $i$ then its arrival time is taken to be zero.

If a commodity $k$ arrives at seaport $i$ before loading starts for its service, then it is stocked at the seaport until the start time of loading for the service that it uses to leave seaport $i$. Constraints (8) and (14) compute the amount of time for which commodity $k$ is stocked at seaport $i$.

Constraints (9) ensure that the commodities arrive before the cutoff time of the service they would like to use and that they arrive at their destinations no later than the duedates. Finally, constraints (10) avoid direct shipments by trucks if the commodity cannot be on time using this transportation mode. These constraints can be dropped by setting $y^{k}=0$ for commodities $k \in K$ such that $r_{k}+\tau_{o_{k}} d_{k}>q_{k}$.

The objective function (1) is the sum of the cost of transportation and the cost of stocking at seaports.

Several problem variations can be modeled as follows.

In the above problem definition, we assume that commodities are picked up at their origins at their release times. Hence a commodity that uses maritime services arrives at the first seaport $i$ at time $r_{k}+\tau_{o_{k} i}$. If this time is earlier than the start time of the maritime service that this commodity uses, then a stocking cost is incurred. However, some clients may have depots where they can stock their goods until the pickup time at no cost. In that case, it may be cheaper to pickup these commodities later than their release times. Let $K^{\prime}$ be the set of such commodities. To model this variant, we replace constraints (8) with constraints

$$
z_{i}^{k} \geq \sum_{a \in A: s(a)=i} e_{a} x_{a}^{k}-v_{i}^{k} \quad \forall k \in K \backslash K^{\prime}, i \in N
$$




$$
z_{i}^{k} \geq \sum_{a \in A: s(a)=i} e_{a} x_{a}^{k}-v_{i}^{k}-M x_{o(k) i} \quad \forall k \in K^{\prime}, i \in N
$$

where $M$ is a large number. Constraints (15) are the same as constraints (8) and model the waiting times for commodities that should be picked up at their release times. Constraints (16) are for clients that have depots to stock their goods until their pickup times. These constraints ensure that the waiting time at seaport $i \in N$ is bounded below by a nonpositive number if $i$ is the first seaport that commodity $k \in K^{\prime}$ visits on its path. If $i$ is not the first seaport, i.e., if $x_{o(k) i}=0$, then these constraints are the same as (15).

To model situations where the departure times of maritime services are later than the cutoff times, we replace constraints (7) with constraints

$$
v_{i}^{k}=\sum_{a \in A: t_{a}=i}\left(b_{a}+\tau_{a}\right) x_{a}^{k}+\left(r_{k}+\tau_{o_{k} i}\right) x_{o_{k} i}^{k} \quad \forall k \in K, i \in N
$$

where $b_{a}$ denotes the departure time for maritime service $a \in A$.

In our model, we assume that the stocking penalties are linear functions of the waiting time. We can easily model the situation where there is a period of stocking free of charge and there is a limit on waiting times. Suppose that at seaport $i \in N$, it is possible to stock goods at no cost for a period of length $\chi_{i}$, after which a cost of $\pi_{i}$ units is charged per unit volume and unit time and the stocking period cannot be longer than $v_{i}$ units of time. We can model these requirements by introducing the variables $\eta_{i}^{k}$ for all $k \in K$ and $i \in N$, adding the constraints

$$
\begin{array}{ll}
\eta_{i}^{k} \geq z_{i}^{k}-\chi_{i} & \forall k \in K, i \in N \\
z_{i}^{k} \leq v_{i} & \forall k \in K, i \in N \\
\eta_{i}^{k} \geq 0 & \forall k \in K, i \in N,
\end{array}
$$

and replacing the objective function by

$$
\min \sum_{k \in K} w_{k}\left(\sum_{i \in N} c_{o_{k} i} x_{o_{k} i}^{k}+\sum_{a \in A} c_{a} x_{a}^{k}+\sum_{i \in N} c_{i d_{k}} x_{i d_{k}}^{k}+c_{o_{k} d_{k}} y^{k}+\sum_{i \in N} \pi_{i} \eta_{i}^{k}\right) .
$$

For those seaports $i \in N$ for which the stocking cost is positive, i.e., $\pi_{i}>0$, in an optimal solution $\eta_{i}^{k}=\left(z_{i}^{k}-\chi_{i}\right)^{+}$, and hence no stocking cost is paid for the first $\chi_{i}$ units of the waiting time, and a stocking cost is charged for each unit of extra waiting time.

\section{Variable fixing and valid inequalities}

In this section, we first present a simple result, which enables us to fix the values of some of the variables. Then we derive some valid inequalities based on time restrictions.

Let $F$ denote the set of feasible solutions to model $M 1$. 


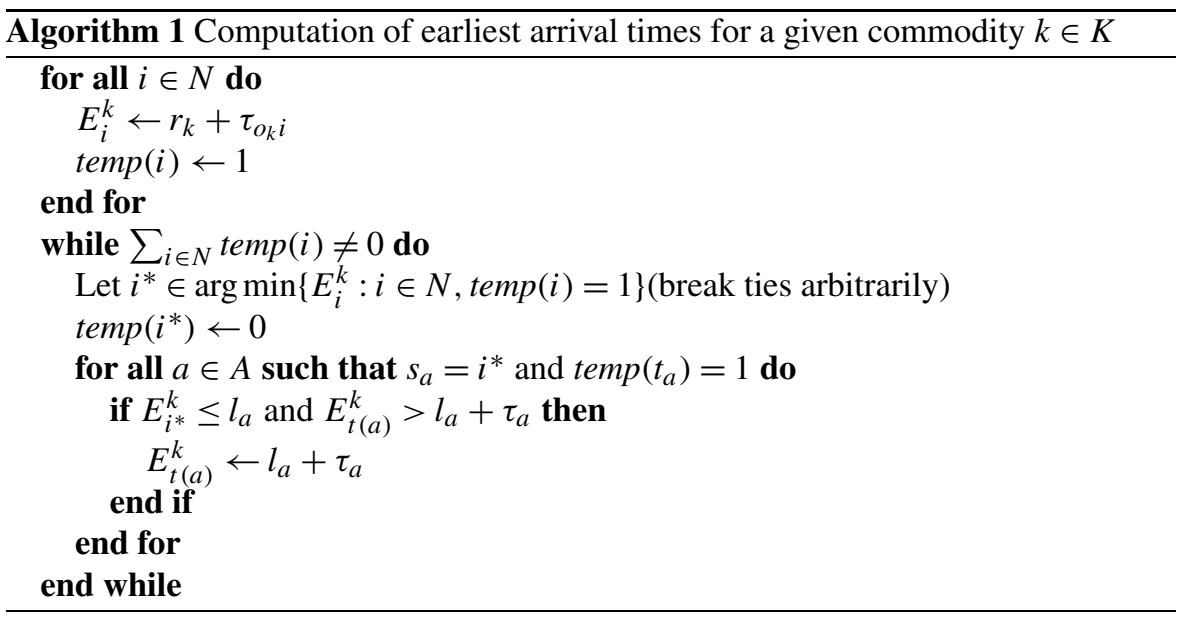

For each commodity $k \in K$ and node $i \in N$, let $E_{i}^{k}$ be the earliest time at which commodity $k$ can arrive at node $i$. These values can be computed using Algorithm 1 , which is a modification of Dijkstra's algorithm (see [1]). Let $k \in K$. The algorithm starts with assigning the value $r_{k}+\tau_{o_{k} i}$ to $E_{i}^{k}$ for all $i \in N$. At each iteration, a list of temporarily labeled nodes is kept. Initially the set of temporarily labeled nodes is equal to the set of nodes. A temporarily labeled node, say $i^{*}$, with the smallest $E_{i}^{k}$ value is chosen as the next permanently labeled node. Then, for each service $a$ that starts at node $i^{*}$, ends at a temporarily labeled node $t(a)$, and whose cutoff time is not earlier than $E_{i^{*}}^{k}$, we compute $l_{a}+\tau_{a}$. This is the time we arrive at the destination seaport $t(a)$ using maritime service $a$. If this value is less than the current value of $E_{t_{a}}^{k}$, then we update $E_{t_{a}}^{k}$ as $l_{a}+\tau_{a}$. In other words, for $a \in A$ with $s_{a}=i^{*}$, $t$ (a) temporarily labeled, and $E_{i^{*}}^{k} \leq l_{a}$, we update $E_{t_{a}}^{k}$ with $\min \left\{E_{t_{a}}^{k}, l_{a}+\tau_{a}\right\}$. The algorithm stops when no temporarily labeled node is left.

If the demand volume of a commodity exceeds the capacity of a service or if the earliest time at which this commodity can arrive at the origin seaport of a service is later than the cutoff time for that service, then clearly, this commodity cannot use that service. Hence, we have the following result:

Proposition 2 Let $k \in K$ and $a \in A$. Every feasible solution satisfies $x_{a}^{k}=0$ if $w_{k}>$ $u_{a}$ or $E_{s_{a}}^{k}>l_{a}$.

Consider commodity $k \in K$ and seaport $i \in N$. If commodity $k$ travels directly from its origin node $o_{k}$ to seaport $i$, then it arrives there at time $r_{k}+\tau_{o_{k} i}$. It is not possible for this commodity to use any service for which the origin seaport is node $i$ and the cutoff time is earlier than $r_{k}+\tau_{o_{k} i}$. The set $O_{i}^{k}=\left\{a \in A: s_{a}=i, r_{k}+\tau_{o_{k} i}>\right.$ $l_{a}$ \} is the set of such services.

Similarly, if commodity $k$ travels from seaport $i$ directly to its destination $d_{k}$, then it should be ready to depart from $i$ the latest at time $q_{k}-\tau_{i d_{k}}$. Hence any service $a$ that arrives at seaport $i$ later than this time cannot be used by commodity $k$. The set of such infeasible services is $D_{i}^{k}=\left\{a \in A: t_{a}=i, l_{a}+\tau_{a}+\tau_{i d_{k}}>q_{k}\right\}$. 
Proposition 3 For $k \in K$ and $i \in N$, the inequalities

$$
x_{o_{k} i}^{k}+\sum_{a \in O_{i}^{k}} x_{a}^{k} \leq 1-y^{k}
$$

and

$$
x_{i d_{k}}^{k}+\sum_{a \in D_{i}^{k}} x_{a}^{k} \leq 1-y^{k}
$$

are valid for $F$.

Proof We give the proof for inequality (17). For commodity $k \in K$, if $y^{k}=1$, then commodity $k$ is transported from its origin to its destination directly on trucks and hence all associated $x$ variables are zero. Otherwise, if $x_{o_{k} i}^{k}=1$, then by definition of set $O_{i}^{k}$, commodity $k$ cannot use any service in this set and hence $\sum_{a \in O_{i}^{k}} x_{a}^{k}=0$. Finally, if $y^{k}=0$ and $x_{o_{k} i}^{k}=0$, then $\sum_{a \in O_{i}^{k}} x_{a}^{k} \leq 1$ as commodity $k$ has to be carried on a simple path. The proof for inequality (18) is similar.

For $a \in A$, define $\Delta_{a}^{+}=\left\{a^{\prime}: s_{a^{\prime}}=t_{a}, l_{a}+\tau_{a}>l_{a^{\prime}}\right\}$ and $\Delta_{a}^{-}=\left\{a^{\prime}: t_{a^{\prime}}=t_{a}, l_{a^{\prime}}+\right.$ $\left.\tau_{a^{\prime}} \geq l_{a}+\tau_{a}\right\}$. Observe that if a commodity $k$ uses service $a$, then the time it reaches the destination seaport $t_{a}$ is $l_{a}+\tau_{a}$. If $a^{\prime}$ is a service that starts at node $t_{a}$ and if the cutoff time for this service is earlier than $l_{a}+\tau_{a}$, then the commodity cannot use this service together with service $a$. Hence, the set $\Delta_{a}^{+}$is the set of services that start at node $t_{a}$ and that cannot be used if service $a$ is used to reach node $t_{a}$. The set $\Delta_{a}^{-}$is the set of services that arrive at node $t_{a}$ not earlier than service $a$.

Proposition 4 Let $k \in K$ and $a \in A$.

If $r_{k}+\tau_{o_{k} t_{a}} \leq l_{a}+\tau_{a}$ and $l_{a}+\tau_{a}+\tau_{t_{a} d_{k}} \leq q_{k}$, the inequality

$$
\sum_{a^{\prime} \in \Delta_{a}^{-}} x_{a^{\prime}}^{k}+\sum_{a^{\prime} \in \Delta_{a}^{+}} x_{a^{\prime}}^{k} \leq 1-y^{k}
$$

is valid for $F$.

If $r_{k}+\tau_{o_{k} t_{a}}>l_{a}+\tau_{a}$ and $l_{a}+\tau_{a}+\tau_{t_{a} d_{k}} \leq q_{k}$, the inequality

$$
x_{o_{k} t_{a}}^{k}+\sum_{a^{\prime} \in \Delta_{a}^{-}} x_{a^{\prime}}^{k}+\sum_{a^{\prime} \in \Delta_{a}^{+}} x_{a^{\prime}}^{k} \leq 1-y^{k}
$$

is valid for $F$.

If $r_{k}+\tau_{o_{k} t_{a}} \leq l_{a}+\tau_{a}$ and $l_{a}+\tau_{a}+\tau_{t_{a} d_{k}}>q_{k}$, the inequality

$$
\sum_{a^{\prime} \in \Delta_{a}^{-}} x_{a^{\prime}}^{k}+\sum_{a^{\prime} \in \Delta_{a}^{+}} x_{a^{\prime}}^{k}+x_{t_{a} d_{k}}^{k} \leq 1-y^{k}
$$

is valid for $F$. 


$$
\begin{aligned}
& \text { If } r_{k}+\tau_{o_{k} t_{a}}>l_{a}+\tau_{a} \text { and } l_{a}+\tau_{a}+\tau_{t_{a} d_{k}}>q_{k} \text {, the inequality } \\
& \qquad x_{o_{k} t_{a}}^{k}+\sum_{a^{\prime} \in \Delta_{a}^{-}} x_{a^{\prime}}^{k}+\sum_{a^{\prime} \in \Delta_{a}^{+}} x_{a^{\prime}}^{k}+x_{t_{a} d_{k}}^{k} \leq 1-y^{k}
\end{aligned}
$$

is valid for $F$.

Proof We give the proof for inequality (22). If $y^{k}=1$, then the vector $x^{k}$ is a zero vector. If $y^{k}=0$ and $x_{a}^{k}=1$, then $\sum_{a^{\prime} \in \Delta_{a}^{+}} x_{a^{\prime}}^{k}=0$ since service $a$ arrives at seaport $t_{a}$ later than the cutoff time for these services, $x_{t_{a} d_{k}}^{k}=0$ since it is not possible to meet the duedate as $l_{a}+\tau_{a}+\tau_{t_{a} d_{k}}>q_{k}, x_{o_{k} t_{a}}^{k}+\sum_{a^{\prime} \in \Delta_{a}^{-} \backslash\{a\}} x_{a^{\prime}}^{k}=0$ since commodity $k$ arrives at seaport $t_{a}$ using service $a$ and cannot use any other service that ends at the same seaport and cannot be carried to this seaport from its origin. Finally, if $y^{k}=0$ and $x_{a}^{k}=0$, then we know that $x_{o_{k} t_{a}}^{k}+\sum_{a^{\prime} \in \Delta_{a}^{-}} x_{a^{\prime}}^{k} \leq 1$ and $\sum_{a^{\prime} \in \Delta_{a}^{+}} x_{a^{\prime}}^{k}+x_{t_{a} d_{k}}^{k} \leq 1$ since commodity $k$ can arrive at and leave seaport $t_{a}$ at most once. Moreover, all services in set $\Delta_{a}^{-}$arrive at $t_{a}$ too late to be able to use any service from set $\Delta_{a}^{+}$or for the commodity to be delivered to its destination on time. Hence at most one of the sums $x_{o_{k} t_{a}}^{k}+\sum_{a^{\prime} \in \Delta_{a}^{-}} x_{a^{\prime}}^{k}$ and $\sum_{a^{\prime} \in \Delta_{a}^{+}} x_{a^{\prime}}^{k}+x_{t_{a} d_{k}}^{k}$ can be 1 .

Before concluding this section, we note that an alternative formulation can be obtained by dropping constraints (9) and using inequalities (17)-(22). But these inequalities do not necessarily imply (9) in the linear programming relaxation. Hence, we keep (9) in our model when we add valid inequalities (17)-(22) to obtain a stronger formulation.

\section{Lagrangian relaxation and an extended formulation}

In the first model given above, the only constraints that link different commodities together are the capacity constraints (6). Next, we investigate a Lagrangian relaxation where these constraints are dualized. Let $\alpha_{a}$ be the Lagrange multiplier associated with constraint (6) for service $a \in A$. For a given vector $\alpha \geq 0$, the relaxed problem disaggregates into $|K|$ problems as follows.

$$
\begin{aligned}
L R(\alpha)= & -\sum_{a \in A} \alpha_{a} u_{a}+\sum_{k \in K: r_{k}+\tau_{o_{k} d_{k}} \leq q_{k}} w_{k} \min \left\{L R^{k}(\alpha), c_{o_{k} d_{k}}\right\} \\
& +\sum_{k \in K: r_{k}+\tau_{o_{k} d_{k}}>q_{k}} w_{k} L R^{k}(\alpha)
\end{aligned}
$$

where

$$
\begin{aligned}
L R^{k}(\alpha)= & \min \\
\text { s.t. } & \sum_{i \in N} c_{o_{k} i} x_{o_{k} i}^{k}+\sum_{a \in A}\left(c_{a}+\alpha_{a}\right) x_{a}^{k}+\sum_{i \in N}^{k} c_{i d_{k}} x_{i d_{k}}^{k}+\sum_{i \in N} p_{i} z_{i}^{k} \\
& \sum_{i \in N}
\end{aligned}
$$




$$
\begin{aligned}
& \sum_{a \in A: t_{a}=i} x_{a}^{k}+x_{o_{k} i}^{k}-\sum_{a \in A: s_{a}=i} x_{a}^{k}-x_{i d_{k}}^{k}=0 \quad \forall i \in N \\
& \sum_{i \in N} x_{i d_{k}}^{k}=1 \\
& \sum_{a \in A: t_{a}=i} x_{a}^{k}+x_{o_{k} i}^{k} \leq 1 \quad \forall i \in N \\
& v_{i}^{k}=\sum_{a \in A: t_{a}=i}\left(l_{a}+\tau_{a}\right) x_{a}^{k}+\left(r_{k}+\tau_{o_{k}} i\right) x_{o_{k} i}^{k} \quad \forall i \in N \\
& z_{i}^{k} \geq \sum_{a \in A: s_{a}=i} e_{a} x_{a}^{k}-v_{i}^{k} \quad \forall i \in N \\
& v_{i}^{k} \leq \sum_{a \in A: s_{a}=i} l_{a} x_{a}^{k}+\left(q_{k}-\tau_{i d_{k}}\right) x_{i d_{k}}^{k} \quad \forall i \in N \\
& x_{o_{k} i}^{k}, x_{i d_{k}}^{k} \in\{0,1\} \quad \forall i \in N \\
& x_{a}^{k} \in\{0,1\} \quad \forall a \in A \\
& z_{i}^{k} \geq 0 \quad \forall i \in N .
\end{aligned}
$$

For a given commodity $k \in K$, the aim of the above problem is to find a time feasible intermodal path from the origin of commodity $k$ to its destination that minimizes the sum of transportation and stocking costs using scheduled services for a unit flow. The cost of using service $a \in A$ is equal to $c_{a}+\alpha_{a}$ in this problem.

If it is feasible to route commodity $k$ directly from its origin to its destination, i.e., if $r_{k}+\tau_{o_{k} d_{k}} \leq q_{k}$, then to compute the contribution of commodity $k$ to the objective function value of the relaxed problem, we take the minimum cost choice among a best intermodal route and the direct shipment. If $r_{k}+\tau_{o_{k} d_{k}}>q_{k}$, then the contribution of commodity $k$ to the objective function value of the relaxed problem is equal to the routing and stocking costs on a best intermodal route computed solving the related subproblem.

In the sequel, we assume that cycles do not occur due to the cost structure. For a given commodity $k \in K$, the above subproblem can be solved using a shortest path algorithm on an acyclic graph. For $i \in N$, let $I_{i}=\left\{a \in A: t_{a}=i\right\}$ be the set of services for which $i$ is the destination seaport and $\Gamma_{i}^{k}=\bigcup_{a \in I_{i}}\left\{l_{a}+\tau_{a}\right\} \cup\left\{r_{k}+\tau_{o_{k} i}\right\}$ be the set of possible arrival times at node $i$. Observe that the size of set $\Gamma_{i}^{k}$ is at most the number of incoming arcs of node $i$ in the original graph $G$ plus 1 . Define an auxiliary graph $G_{k}=\left(N_{k}, A_{k}\right)$ where $N_{k}=\left\{o_{k}, d_{k}\right\} \cup\left\{(i, j): i \in N, j \in \Gamma_{i}^{k}\right\}$, $O_{k}=\left\{\left(o_{k},\left(i, r_{k}+\tau_{o_{k} i}\right)\right): i \in N\right\}, D_{k}=\left\{\left((i, j), d_{k}\right):(i, j) \in N_{k}, j+\tau_{i d_{k}} \leq q_{k}\right\}$, $S_{k}=\left\{\left(\left(i_{1}, j_{1}\right),\left(i_{2}, j_{2}\right)\right):\left(i_{1}, j_{1}\right),\left(i_{2}, j_{2}\right) \in N_{k}, \exists a \in A: s_{a}=i_{1}, t_{a}=i_{2}, j_{1} \leq l_{a}, l_{a}+\right.$ $\left.\tau_{a}=j_{2}\right\}$, and $A_{k}=O_{k} \cup D_{k} \cup S_{k}$. The node set $N_{k}$ includes the origin and destination of commodity $k$ and one node for each seaport $i$ and a possible arrival time at $i$. The arc set $O_{k}$ consists of all arcs from the origin to nodes $\left(i, r_{k}+\tau_{o_{k} i}\right)$, which represent the seaports and the associated arrival times from the origin using trucks. The arc set $D_{k}$ consists of all arcs from nodes $(i, j)$ to the destination $d_{k}$ such that it is possible 
to arrive at the destination by the duedate if we leave seaport $i$ using trucks at time $j$. Finally, the arc set $S_{k}$ consists of arcs from node $\left(i_{1}, j_{1}\right)$ to node $\left(i_{2}, j_{2}\right)$ such that there exists a maritime service from seaport $i_{1}$ to seaport $i_{2}$ whose cutoff time is not earlier than $j_{1}$ and whose arrival time at seaport $i_{2}$ is equal to $j_{2}$. In other words, arc $\left(\left(i_{1}, j_{1}\right),\left(i_{2}, j_{2}\right)\right)$ is in set $S_{k}$ if and only if it is possible to travel from seaport $i_{1}$ to seaport $i_{2}$ using a maritime service that arrives at seaport $i_{2}$ at time $j_{2}$ if we arrive at seaport $i_{1}$ at time $j_{1}$.

First remark that graph $G_{k}$ does not contain parallel arcs. Second, the number of nodes in this graph is in the order of the number of maritime services, i.e., $O(|A|)$. Third, if we assume that all travel times are positive, then the graph $G_{k}$ is acyclic and can be lexicographically ordered by sorting the nodes $(i, j)$ in nondecreasing order of $j$.

Consider a path from node $o_{k}$ to node $d_{k}$ in this graph. If this path uses an arc $\left(\left(i_{1}, j_{1}\right),\left(i_{2}, j_{2}\right)\right)$, then the commodity arrives at seaport $i_{1}$ at time $j_{1}$ and then travels directly to seaport $i_{2}$ and arrives there at time $j_{2}$. As this arc exists in graph $G_{k}$, we know that there exists at least one maritime service that departs from seaport $i_{1}$ and arrives at the destination seaport $i_{2}$ at time $j_{2}$ and for which the cutoff time is not earlier than $j_{1}$. Hence a path from node $o_{k}$ to node $d_{k}$ in graph $G_{k}$ is a path from node $o_{k}$ to node $d_{k}$ that satisfies all time restrictions.

Next, we define the lengths of the arcs. The length of $\operatorname{arc}\left(o_{k},\left(i, r_{k}+\tau_{o_{k}} i\right)\right) \in O_{k}$ is equal to $c_{o_{k} i}$ and the length of arc $\left((i, j), d_{k}\right) \in D_{k}$ is equal to $c_{i d_{k}}$. Finally, the length of an $\operatorname{arc}\left(\left(i_{1}, j_{1}\right),\left(i_{2}, j_{2}\right)\right) \in S_{k}$ is equal to $\min _{a \in A: s_{a}=i_{1}, t_{a}=i_{2}, j_{1} \leq l_{a}, l_{a}+\tau_{a}=j_{2}}\left(c_{a}+\alpha_{a}+\right.$ $\left.p_{i_{1}}\left(e_{a}-j_{1}\right)^{+}\right)$. Here we look at all maritime services that travel from seaport $i_{1}$ to seaport $i_{2}$, whose cutoff times are not earlier than $j_{1}$ and whose arrival times are $j_{2}$. If $a$ is such a service and if the commodity uses this service, then it waits at seaport $i_{1}$ until the start time of loading, i.e., for $\left(e_{a}-j_{1}\right)^{+}$units of time.

Song and Chen [20] use a graph generation procedure similar to ours. The difference is that they use both arrival and departure times in defining their nodes, whereas we only use arrival times.

Now $L R^{k}(\alpha)$ is equal to the length of a shortest path from the origin $o_{k}$ to the destination $d_{k}$ in graph $G_{k}$. As the size of the graph $G_{k}$ grows polynomially with the size of the problem, $L R(\alpha)$ can be computed efficiently.

The Lagrangian dual bound is $L D=\max _{\alpha \geq 0} L R(\alpha)$. This bound is at least as good as the linear programming bound of model $M l$. As the subproblems are shortest path problems, we can derive an extended formulation whose linear programming relaxation yields the same bound as the Lagrangian dual.

For each commodity $k \in K$, we define a graph where a simple path from origin $o_{k}$ to destination $d_{k}$ defines a trip that starts at $o_{k}$, ends at $d_{k}$, and respects all time restrictions. We define the costs of arcs on the new graph in such a way that the sum of costs of arcs on a path is equal to the transportation and stocking cost for the corresponding trip. The problem $I M R-S$, then, is equivalent to the problem of finding a path for each commodity $k$ such that the capacity constraints are satisfied.

For each $k \in K$, we define the following graph $G_{k}^{\prime}=\left(N_{k}, A_{k}^{\prime}\right)$. The set $A_{k}^{\prime}$ consists of arcs of $O_{k}$ and $D_{k}$ and a set of service arcs $S_{k}^{\prime}$ defined as follows. For each service $a \in A$ and nodes $\left(i_{1}, j_{1}\right)$ and $\left(i_{2}, j_{2}\right)$ in $N_{k}$ such that $s_{a}=i_{1}, t_{a}=i_{2}, j_{1} \leq l_{a}$ and $l_{a}+\tau_{a}=j_{2}$, we add an arc $\omega$ from node $\left(i_{1}, j_{1}\right)$ to node $\left(i_{2}, j_{2}\right)$ with cost $\sigma_{\omega}=$ 
$c_{a}+p_{i_{1}}\left(e_{a}-j_{1}\right)^{+}$. In other words, if it is possible to go from seaport $i_{1}$ to seaport $i_{2}$ using maritime service $a$ that leaves $i_{1}$ at a time not earlier than $j_{1}$ and arrives at $i_{2}$ at time $j_{2}$, then we add arc $\omega$. The unit cost associated with arc $\omega$ is equal to the unit transportation cost using maritime service $a$ plus the cost of waiting at seaport $i_{1}$ from time $j_{1}$ until time $e_{a}$, the start time of loading for service $a$.

We define $\rho(\omega)=a$. For arc $\omega=\left(o_{k},\left(i, r_{k}+\tau_{o_{k}} i\right)\right) \in O_{k}$, we define $\sigma_{\omega}=c_{o_{k}} i$ and for $\operatorname{arc} \omega=\left((i, j), d_{k}\right) \in D_{k}$, we define $\sigma_{\omega}=c_{i d_{k}}$.

Note that different from graph $G_{k}$, in graph $G_{k}^{\prime}$ we may have parallel arcs with different costs and/or capacities. Still, the number of arcs remains polynomial in the number of maritime services.

For the extended formulation, we define the following additional variables. Let $k \in K$. For arc $\omega=\left(o_{k},\left(i, r_{k}+\tau_{o_{k} i}\right)\right) \in O_{k}$, let $f_{\omega}^{k}$ be 1 if commodity $k$ travels directly from its origin to seaport $i$ and arrives there at time $r_{k}+\tau_{o_{k} i}$ and 0 otherwise. For arc $\omega=\left((i, j), d_{k}\right) \in D_{k}$, let $f_{\omega}^{k}$ be 1 if commodity $k$ arrives at seaport $i$ at time $j$ and travels from $i$ directly to its destination and 0 otherwise. Finally, for arc $\omega \in S_{k}^{\prime}$ from node $\left(i_{1}, j_{1}\right)$ to $\left(i_{2}, j_{2}\right)$, we define $f_{\omega}^{k}$ to be 1 if commodity $k$ arrives at seaport $i_{1}$ at time $j_{1}$ and uses service $\rho(\omega)$, which departs from seaport $i_{1}$ and arrives at seaport $i_{2}$ at time $j_{2}$ and 0 otherwise.

Let $\delta^{-}(k, i 1, j 1)$ and $\delta^{+}(k, i 1, j 1)$ be the sets of incoming and outgoing arcs of node $\left(i_{1}, j_{1}\right) \in N_{k}$ in graph $G_{k}^{\prime}$.

The extended formulation, $M 2$ is as follows.

$$
\begin{array}{ll}
\min & \sum_{k \in K} w_{k}\left(\sum_{\omega \in A_{k}} \sigma_{\omega} f_{\omega}^{k}+c_{o_{k} d_{k}} y^{k}\right) \\
\text { s.t. } & \sum_{\omega=\left(o_{k},\left(i, r_{k}+\tau_{o} i\right)\right) \in O_{k}} f_{\omega}^{k}+y^{k}=1 \quad \forall k \in K \\
& \sum_{\omega \in \delta^{+}(k, i 1, j 1)} f_{\omega}^{k}-\sum_{\omega \in \delta^{-}(k, i 1, j 1)} f_{\omega}^{k}=0 \quad \forall k \in K,\left(i_{1}, j_{1}\right) \in N_{k} \\
& \sum_{\omega=\left((i, j), d_{k}\right) \in D_{k}} f_{(i, j), d_{k}}^{k}+y^{k}=1 \quad \forall k \in K \\
& \sum_{k \in K} w_{k} \sum_{\omega \in S_{k}^{\prime}: \rho(\omega)=a} f_{\omega}^{k} \leq u_{a} \quad \forall a \in A \\
& r_{k}+\tau_{o_{k} d_{k}} y^{k} \leq q_{k} \quad \forall k \in K \\
& f_{\omega}^{k} \in\{0,1\} \quad \forall k \in K, \omega \in A_{k}^{\prime} \\
& y^{k} \in\{0,1\} \quad \forall k \in K .
\end{array}
$$

Constraints (35)-(37) and (39)-(41) ensure that each commodity is routed on a path that satisfies all time related constraints. Constraints (38) are the capacity constraints for the maritime services.

Here we remark that model $M 2$ uses a time-space network to model time related constraints. However, unlike most models based on time-space networks, the size of 
model M2 does not depend on the length of the planning horizon and is polynomial in the number of commodities and number of services. This is due to the fact that the services are scheduled and it is sufficient to consider only the arrival times at seaports in generating the time-space network.

In the above formulation, if we define the direct shipments using $\operatorname{arcs}\left(o_{k}, d_{k}\right)$, then we obtain an origin-destination integer multicommodity flow problem (see, e.g., Barnhart et al. [3]).

To conclude this section, we compare the strength of bounds we can obtain from the linear programming relaxations of the models with and without variable fixing and valid inequalities and the Lagrangian dual bound.

Let $M 1 T$ be model $M 1$ together with the constraints $x_{a}^{k}=0$ for all $k \in K$ and $a \in A$ with $E_{s_{a}}^{k}>l_{a}$ and inequalities (17)-(22). In other words, $M 1 T$ is model $M I$ strengthened with variable fixing and valid inequalities based on time restrictions. Let $M 1 T C$ be model $M 1 T$ together with the constraints $x_{a}^{k}=0$ for all $k \in K$ and $a \in A$ with $w_{k}>u_{a}$, i.e., the model $M 1$ strengthened with variable fixing and valid inequalities based on time restrictions and capacity constraints.

Let $M 2 C$ be model $M 2$ together with the constraints $\sum_{\omega \in S_{k}^{\prime}: \rho(\omega)=a} f_{\omega}^{k}=0$ for all $k \in K$ and $a \in A$ with $w_{k}>u_{a}$. Finally let $L D C$ be the Lagrangian dual bound when the same Lagrangian relaxation is applied to model $M I$ together with the constraints $x_{a}^{k}=0$ for all $k \in K$ and $a \in A$ with $w_{k}>u_{a}$. Note that these additional constraints can be handled by ignoring the corresponding services in constructing graph $G_{k}$.

For a given model $M$, let $L P(M)$ be the optimal value of its linear programming relaxation. The following theorem gives a comparison of the linear programming bounds of several models and the Lagrangian Dual.

Theorem $1 L D=L P(M 2) \geq L P(M 1 T)$ and $L D C=L P(M 2 C) \geq L P(M 1 T C)$.

Proof We give the proof for $L D=L P(M 2) \geq L P(M 1 T)$.

We first prove that the Lagrangian dual bound $L D$ is equal to the linear programming bound of $M 2$.

First observe that $L R(\alpha)=-\sum_{a \in A} \alpha_{a} u_{a}+\sum_{k \in K} w_{k} \overline{L R}^{k}(\alpha)$ where

$$
\begin{gathered}
\overline{L R}^{k}(\alpha)=\min \sum_{\omega=\left(o_{k},\left(i, r_{k}+\tau_{o_{k}}\right)\right) \in O_{k}} \sigma_{\omega} f_{\omega}^{k}+\sum_{\omega \in S_{k}^{\prime}}\left(\sigma_{\omega}+\alpha_{\rho(\omega)}\right) f_{\omega}^{k} \\
+\sum_{\omega=\left((i, j), d_{k}\right) \in D_{k}} \sigma_{\omega} f_{\omega}^{k}+c_{o_{k} d_{k} y^{k}} \\
\text { s.t. } \quad \sum_{\omega=\left(o_{k},\left(i, r_{k}+\tau_{o_{k}}\right)\right) \in O_{k}} f_{\omega}^{k}+y^{k}=1 \\
\sum_{\omega \in \delta^{+}(k, i 1, j 1)} f_{\omega}^{k}-\sum_{\omega \in \delta^{-}(k, i 1, j 1)} f_{\omega}^{k}=0 \quad \forall\left(i_{1}, j_{1}\right) \in N_{k} \\
\sum_{\omega=\left((i, j), d_{k}\right) \in D_{k}} f_{\omega}^{k}+y^{k}=1
\end{gathered}
$$




$$
\begin{aligned}
& f_{\omega}^{k} \in\{0,1\} \quad \forall \omega \in A_{k}^{\prime} \\
& y^{k} \in\{0,1\} .
\end{aligned}
$$

As the above problem is a shortest path problem, the convex hull of its feasible solutions is described by constraints (42)-(44) and nonnegativity constraints on variables. Hence the Lagrangian dual bound $L D$ is equal to the linear programming bound of M2 (see, e.g., Wolsey [21]).

Now suppose that we apply the Lagrangian relaxation to the model MIT. As the constraints $x_{a}^{k}=0$ for all $k \in K$ and $a \in A$ with $E_{s_{a}}^{k}>l_{a}$ and inequalities (17)-(22) are redundant in the relaxed problem, we have that $L D \geq L P(M 1 T)$.

The second statement can be proved in a similar way.

\section{Computational results}

In this chapter, we first describe the test data and then present the results obtained by solving our integer models.

We use two service networks in our computational study. The first one is the network of the third party logistics company mentioned in the Introduction. This network includes 34 important seaports in Europe, Russia, China, Hong Kong, Singapore, and Malaysia and 167 services of the world's major sea transportation operators. The schedules of services are obtained from the company. These schedules are for the services provided in March 2008. The other parameters we obtained from the company are the costs and capacities.

We named the company's network as $\mathrm{O} 1$. We also generated a random network $\mathrm{O} 2$ with 66 nodes and 1200 arcs. For the instances on network O1, we randomly generate the demands. For the other instances, we generate all data randomly.

For each service network, we generate 4 core instances with different number of commodities, namely, $|K|$ is equal to $400,600,800$, and 1000 .

For each core instance, we multiply the arc capacities by a capacity factor to create three different instances with tight, medium, and loose capacities. The capacity factor takes the values $2 / 3,1$, and 2 . As a result, we have 24 problem instances. An instance is named as follows: name of the service network-capacity factor-number of commodities.

We solve our models using CPLEX 11.0. All runs are taken on a $2.67 \mathrm{GHz}$ $2 \times$ Quadcore Xeon Processor with 8 GB Ram.

To compare the performances of the two models and investigate the effect of variable fixing and valid inequalities, we solve each problem instance using models $M 1$, $M 1 v$ (the first model with valid inequalities), $M I f$ (the first model with variable fixing), $M 1 T C$ (the first model with variable fixing and valid inequalities), $M 2 C$ (the extended model with variable fixing based on capacity restrictions). For each instance and model, we report the duality gap, i.e., the percentage gap between the optimal value of the integer model and the optimal value of its linear programming relaxation, the number of nodes in the branch and cut tree and the cpu time in seconds. We impose a time limit of one hour. For the instances that are not solved to optimality 
Table 1 The duality gaps for O1 instances

Table 2 The number of nodes for $\mathrm{O} 1$ instances

\begin{tabular}{lrrrrr}
\hline Instance & \multicolumn{1}{l}{$M 1$} & \multicolumn{1}{l}{$M 1 v$} & $M 1 f$ & $M 1 T C$ & $M 2 C$ \\
\hline O1-2/3-400 & 22.52 & 18.12 & 5.64 & 1.72 & 0.53 \\
O1-2/3-600 & 16.35 & 13.63 & 4.92 & 1.76 & 0.40 \\
O1-2/3-800 & 13.40 & 11.23 & 5.11 & 2.41 & 1.00 \\
O1-2/3-1000 & 11.39 & 9.96 & 4.79 & 2.74 & 1.74 \\
O1-1-400 & 19.56 & 12.69 & 12.38 & 2.59 & 1.71 \\
O1-1-600 & 14.73 & 10.27 & 10.45 & 3.90 & 1.68 \\
O1-1-800 & 12.36 & 8.73 & 9.53 & 4.77 & 1.71 \\
O1-1-1000 & 10.44 & 7.81 & 8.18 & 4.82 & 1.73 \\
O1-2-400 & 16.91 & 4.08 & 16.87 & 4.06 & 1.04 \\
O1-2-600 & 13.52 & 4.24 & 13.50 & 4.22 & 0.75 \\
O1-2-800 & 12.59 & 5.10 & 12.55 & 5.08 & 0.81 \\
O1-2-1000 & 11.08 & 5.35 & 11.02 & 5.30 & 0.86 \\
\hline
\end{tabular}

\begin{tabular}{llrlrl}
\hline Instance & \multicolumn{1}{l}{$M 1$} & $M 1 v$ & \multicolumn{1}{c}{$M 1 f$} & $M 1 T C$ & $M 2 C$ \\
\hline O1-2/3-400 & \multicolumn{1}{c}{830} & 0 & 786 & 0 & 0 \\
O1-2/3-600 & - & 0 & - & 0 & 1252 \\
O1-2/3-800 & - & 0 & - & 0 & 5235 \\
O1-2/3-1000 & - & 67 & - & 67 & - \\
O1-1-400 & 1054 & 0 & 992 & 0 & 1520 \\
O1-1-600 & - & 1 & - & 1 & 10195 \\
O1-1-800 & - & 1 & - & 1 & - \\
O1-1-1000 & - & 409 & - & 409 & - \\
O1-2-400 & \multicolumn{1}{l}{2887} & 10 & 2887 & 10 & 2729 \\
O1-2-600 & - & 96 & - & 96 & - \\
O1-2-800 & - & 221 & - & 201 & - \\
O1-2-1000 & - & 1259 & - & 1252 & - \\
\hline
\end{tabular}

in one hour, we report the remaining percentage gap, as reported by the solver, in parenthesis in tables reporting cpu times.

In Tables 1, 2 and 3, we report the duality gaps, the number of nodes, and the cpu times for the instances on $\mathrm{O} 1$ network, respectively. We report the number of nodes for the instances and models for which optimality could be proved in one hour.

In Table 1, we observe that with model $M 1$, the duality gaps decrease as the number of commodities and the capacity factor increase. The effect of variable fixing is stronger for the problems with tight capacities. This is expected as one of the variable fixing rules is based on capacity constraints. The addition of valid inequalities results in higher improvements for problems with larger capacity factors. After variable fixing and the addition of valid inequalities, the duality gaps are improved significantly but are still much higher compared to the duality gaps of model $M 2 C$. 
Table 3 The cpu times for $\mathrm{O} 1$ instances

\begin{tabular}{llrlrl}
\hline Instance & $M 1$ & $M 1 v$ & $M 1 f$ & $M 1 T C$ & $M 2 C$ \\
\hline O1-2/3-400 & 480.6 & 38.7 & 441.5 & 37.9 & 4.4 \\
O1-2/3-600 & $1 \mathrm{~h}(0.18)$ & 58.0 & $1 \mathrm{~h}(0.16)$ & 57.9 & 42.8 \\
O1-2/3-800 & $1 \mathrm{~h}(0.44)$ & 76.0 & $1 \mathrm{~h}(0.44)$ & 67.4 & 197.9 \\
O1-2/3-1000 & $1 \mathrm{~h}(0.39)$ & 109.3 & $1 \mathrm{~h}(0.38)$ & 108.6 & $1 \mathrm{~h}(0.02)$ \\
O1-1-400 & 812.4 & 47.5 & 779.3 & 38.7 & 18.1 \\
O1-1-600 & $1 \mathrm{~h}(0.58)$ & 80.1 & $1 \mathrm{~h}(0.58)$ & 64.6 & 334.2 \\
O1-1-800 & $1 \mathrm{~h}(18.13)$ & 108.7 & $1 \mathrm{~h}(18.13)$ & 105.6 & $1 \mathrm{~h}(0.05)$ \\
O1-1-1000 & $1 \mathrm{~h}(21.12)$ & 155.8 & $1 \mathrm{~h}(21.10)$ & 194.4 & $1 \mathrm{~h}(0.11)$ \\
O1-2-400 & 1131.4 & 47.4 & 1022.2 & 46.2 & 31.5 \\
O1-2-600 & $1 \mathrm{~h}(1.74)$ & 94.7 & $1 \mathrm{~h}(1.74)$ & 75.5 & $1 \mathrm{~h}(0.13)$ \\
O1-2-800 & $1 \mathrm{~h}(2.44)$ & 137.4 & $1 \mathrm{~h}(2.44)$ & 138.3 & $1 \mathrm{~h}(0.21)$ \\
O1-2-1000 & $1 \mathrm{~h}(4.28)$ & 303.3 & $1 \mathrm{~h}(4.28)$ & 240.9 & $1 \mathrm{~h}(0.24)$ \\
\hline
\end{tabular}

In Tables 2 and 3, we see that only instance with 400 commodities are solved to optimality within one hour of CPU time with model $M 1$. Variable fixing does not change this situation but results in a decrease in the number of nodes evaluated and the solution times for the instances solved to optimality. For a few of the unsolved instances, there is a slight decrease in the final gaps due to variable fixing. However, with both models $M I$ and $M I f$, final gaps are very high for medium capacity factor problems with 800 and 1000 commodities. With formulation $M 2 C$, we can solve larger instances to optimality for small capacity factors. However, for the large capacity factor, only the instance with 400 commodities has been solved to optimality within the timelimit. With this formulation, the remaining gaps is less than $0.25 \%$ for the unsolved problems.

All instances are solved to optimality in less than six minutes when the valid inequalities are added to model $M 1$. For the instances that are solved to optimality with both $M 1 v$ and $M 2 C$, we see that the solver enumerated a smaller number of nodes with $M 1 v$ even though this model results in larger duality gaps. This is probably due to the set packing structure that is created by the valid inequalities and that enables the solver to generate further valid inequalities.

Finally, we observe that even though variable fixing has a significant effect on the duality gaps for some instances, it does not result in an improvement in solution times for those instances.

Next, we report the results obtained for the instances on the randomly generated network O2. This network contains about seven times more arcs than the original graph $\mathrm{O} 1$. The results are given in Tables 4, 5 and 6.

Comparing Tables 1 and 4, we see that the duality gaps are much higher for the random instances with model $M 1$ for the small capacity factor. As in the case of $\mathrm{O} 1$ instances, we see that the effect of valid inequalities is higher for problems with larger capacity factors and the effect of variables fixing is higher for problems with smaller capacity factors. Compared to the gaps with the $\mathrm{O} 1$ instances, with the $\mathrm{O} 2$ instances, 
Table 4 The duality gaps for $\mathrm{O} 2$ instances

Table 5 The number of nodes for $\mathrm{O} 2$ instances

\begin{tabular}{lrrrrr}
\hline Instance & \multicolumn{1}{l}{$M 1$} & \multicolumn{1}{l}{$M 1 v$} & \multicolumn{1}{l}{$M 1 f$} & $M 1 T C$ & $M 2 C$ \\
\hline O2-2/3-400 & 43.48 & 40.47 & 8.78 & 4.08 & 0.84 \\
O2-2/3-600 & 40.22 & 37.27 & 9.72 & 5.07 & 1.28 \\
O2-2/3-800 & 35.55 & 32.02 & 10.31 & 8.26 & 1.82 \\
O2-2/3-1000 & 33.61 & 28.66 & 10.79 & 9.20 & 2.36 \\
O2-1-400 & 15.97 & 10.69 & 14.85 & 10.38 & 0.26 \\
O2-1-600 & 14.91 & 7.59 & 14.22 & 7.41 & 0.31 \\
O2-1-800 & 14.86 & 8.67 & 14.37 & 8.53 & 0.53 \\
O2-1-1000 & 14.85 & 13.01 & 14.48 & 12.91 & 0.50 \\
O2-2-400 & 15.84 & 10.55 & 14.59 & 10.13 & 0.03 \\
O2-2-600 & 14.54 & 7.17 & 13.77 & 6.91 & 0.01 \\
O2-2-800 & 14.35 & 7.80 & 13.79 & 7.61 & 0.01 \\
O2-2-1000 & 14.68 & 8.79 & 14.26 & 8.65 & 0.03 \\
\hline
\end{tabular}

\begin{tabular}{lcrccc}
\hline Instance & $M 1$ & $M 1 v$ & $M 1 f$ & $M 1 T C$ & $M 2 C$ \\
\hline O2-2/3-400 & 1640 & 0 & 1589 & 0 & 0 \\
O2-2/3-600 & - & 0 & - & 0 & 0 \\
O2-2/3-800 & - & 0 & - & 0 & 0 \\
O2-2/3-1000 & - & 0 & - & 0 & 0 \\
O2-1-400 & 69 & 0 & 69 & 0 & 0 \\
O2-1-600 & 495 & 0 & 487 & 0 & 0 \\
O2-1-800 & - & 33 & - & 33 & 0 \\
O2-1-1000 & - & 228 & - & 228 & 0 \\
O2-2-400 & 0 & 0 & 0 & 0 & 0 \\
O2-2-600 & 10 & 0 & 10 & 0 & 0 \\
O2-2-800 & - & 28 & - & 28 & 0 \\
O2-2-1000 & - & 111 & - & 111 & 0 \\
\hline
\end{tabular}

the duality gaps with the first model MITC are higher and the ones with the second model $M 2 C$ are usually smaller.

We could solve larger instances to optimality with model $M 1$ for the $\mathrm{O} 2$ network. Still the instances with 800 and 1000 commodities are not solved to optimality in one hour. The final gaps remain the same after variable fixing. With the addition of valid inequalities, all instances are solved to optimality in maximum six minutes. Model $M 2 C$ could also solve all the instances optimally. From Table 5, we see that these instances are solved at the root node without branching. Even though more nodes are evaluated with model $M I v$, the solution times are shorter compared to the ones with $M 2 C$.

As a summary, we could not solve large instances to optimality with the first model without the valid inequalities. With the inclusion of the valid inequalities, model 1 
Table 6 The CPU times for $\mathrm{O} 2$ instances

\begin{tabular}{llllll}
\hline Instance & $M 1$ & $M 1 v$ & $M 1 f$ & $M 1 T C$ & $M 2 C$ \\
\hline O2-2/3-400 & 240.9 & 111.5 & 232.8 & 101.9 & 272.1 \\
O2-2/3-600 & $1 \mathrm{~h}(0.78)$ & 166.4 & $1 \mathrm{~h}(0.78)$ & 183.7 & 298.2 \\
O2-2/3-800 & $1 \mathrm{~h}(2.58)$ & 231.1 & $1 \mathrm{~h}(2.58)$ & 247.1 & 503.9 \\
O2-2/3-1000 & $1 \mathrm{~h}(4.04)$ & 335.0 & $1 \mathrm{~h}(4.04)$ & 346.2 & 552.4 \\
O2-1-400 & 209.1 & 110.9 & 198.1 & 99.3 & 269.1 \\
O2-1-600 & 3211.9 & 151.2 & 3054.3 & 136.4 & 378.5 \\
O2-1-800 & $1 \mathrm{~h}(1.12)$ & 227.0 & $1 \mathrm{~h}(1.12)$ & 219.2 & 468.2 \\
O2-1-1000 & $1 \mathrm{~h}(2.74)$ & 240.6 & $1 \mathrm{~h}(2.74)$ & 231.5 & 548.1 \\
O2-2-400 & 232.3 & 103.6 & 229.1 & 101.2 & 254.0 \\
O2-2-600 & 667.1 & 162.4 & 663.0 & 160.8 & 362.7 \\
O2-2-800 & $1 \mathrm{~h}(0.60)$ & 188.2 & $1 \mathrm{~h}(0.60)$ & 185.3 & 476.7 \\
O2-2-1000 & $1 \mathrm{~h}(0.84)$ & 418.7 & $1 \mathrm{~h}(0.84)$ & 414.2 & 572.3 \\
\hline
\end{tabular}

outperformed the extended model in terms of computation times and could solve all our instances to optimality in not more than six minutes.

\section{Conclusion}

In this paper, we considered an intermodal multicommodity routing problem with scheduled and capacitated services. We established the computational complexity of the problem and proposed two mixed integer programming formulations. We compared our formulations using Lagrangian relaxation. We strengthened the first formulation with valid inequalities that modeled conflicts due to time related restrictions. Even though the first model strengthened with valid inequalities is still weaker than the second model, it performed better in terms of solution times. We managed to solve instances with 1000 commodities to optimality in maximum six minutes for the two networks considered.

As a further research, we are considering to devise heuristic algorithms based on Lagrangian relaxation. We tested some basic versions of this heuristic on the instances used in the computational study, but we could not obtain acceptable gaps in shorter times than the computation times of the first model with valid inequalities. This was mainly because the subgradient algorithm converged very slowly. We investigate ways to make the convergence faster. We also believe that devising alternative heuristic approaches such as tabu search or variable neighborhood search may be a nice contribution.

Another area of research is to extend the problem proposed in this paper to model more general cost structures and to include higher level decisions, such as the choice of maritime service arcs and their schedules.

Acknowledgements This research is supported by TUBITAK project no. 107M460. 


\section{References}

1. Ahuja, R.K., Magnanti, T.L., Orlin, J.B.: Network Flows. Prentice Hall, New York (1993)

2. Barnhart, C., Ratliff, H.D.: Modeling intermodal routing. J. Bus. Logist. 14, 205-223 (1993)

3. Barnhart, C., Hane, C.A., Vance, P.H.: Using branch-and-price-and-cut to solve origin-destination integer multicommodity flow problems. Oper. Res. 48, 318-326 (2000)

4. Bektas, T., Crainic, T.G.: A brief overview of intermodal transportation. Technical Report, CIRRELT2007-03, Interuniversity Research Centre on Enterprise Networks, Logistics and Transportation (2007)

5. Boardman, B.S., Malstrom, E.M., Butler, D.P., Cole, M.H.: Computer assisted routing of intermodal shipments. Comput. Ind. Eng. 33, 311-314 (1997)

6. Bontekoning, Y.M., Macharis, C., Trip, J.J.: Is a new applied transportation research field emerging?A review of intermodal rail-truck freight transport literature. Transp. Res. A 38, 1-34 (2004)

7. Bookbinder, J.H., Fox, N.S.: Intermodal routing of Canada-Mexico shipments under NAFTA. Transp. Res. E 34, 289-303 (1998)

8. Boussedjra, M., Bloch, C., El Moudni, A.: An exact method to find the intermodal shortest path. In: Proceedings of the IEEE International Conference on Networking, Sensing \& Control, pp. 1075-1080 (2004)

9. Chang, T.S.: Best routes selection in international intermodal networks. Comput. Oper. Res. 35, 28772891 (2008)

10. Christiansen, M., Fagerholt, K., Nygreen, B., Ronen, D.: Maritime transportation. In: Barnhart, C., Laporte, G. (eds.) Handbook in OR \& MS, vol. 14, pp. 189-284. Elsevier, Amsterdam (2007)

11. Crainic, T.G., Kim, K.H.: Intermodal transportation. In: Barnhart, C., Laporte, G. (eds.) Handbook in OR \& MS, vol. 14, pp. 467-537. Elsevier, Amsterdam (2007)

12. Garey, M.R., Johnson, D.S.: Computers and Intractability, A Guide to the Theory of NPCompleteness. Freeman, New York (1979)

13. Grasman, S.E.: Dynamic approach to strategic and operational multimodal routing decisions. Int. J. Logist. Syst. Manag. 2, 96-106 (2006)

14. Kim, H.J., Chang, Y.T., Lee, P.T.T., Shin, S.H., Kim, M.J.: Optimizing the transportation of intermodal container cargoes in Korea. Marit. Policy Manag. 35, 103-122 (2008)

15. Macharis, B., Bontekoning, Y.M.: Opportunities for OR in intermodal freight transportation research: a review. Eur. J. Oper. Res. 153, 400-416 (2004)

16. Min, H.: International intermodal choices via change-constrained goal programming. Transp. Res. A 25, 351-362 (1991)

17. Moccia, L., Cordeau, J.F., Laporte, G., Ropke, S., Valentini, M.P.: Modeling and solving a multimodal routing problem with timetables and time windows. Networks (to appear)

18. Pedersen, M.B.: Optimization models and solution methods for intermodal transportation. PhD Thesis, Technical University of Denmark, Centre for Traffic and Transport (2005)

19. Slack, B.: Intermodal Transportation. In: Brewer, A.M., Button, K.J., Hensher, D.A. (eds.) Handbook of Logistics and Supply-Chain Management, pp. 141-154. Pergamon, Elmsford (2001)

20. Song, H., Chen, G.: Minimum cost delivery problem in intermodal transportation networks. In: Proceedings of the 2007 IEEE IEEM, pp. 1502-1506 (2007)

21. Wolsey, L.A.: Integer Programming. Wiley, New York (1998)

22. Ziliaskopoulos, A., Wardell, W.: An intermodal optimum path algorithm for multimodal networks with dynamic arc travel times and switching delays. Eur. J. Oper. Res. 125, 486-502 (2000) 